\title{
Developing an Aid for Obtaining Visual Eight-Fingered Typing Skill for the General Public
}

\author{
Junichi Watanabe \\ Japan
}

\begin{abstract}
In 1986 when locally designed personal computer (PC) appeared, the author was unexpectedly involved in calamitous key-search difficulty on the PC keyboard. He created a graphic reference table coping with the difficulty. The table reached immediately a visual aid for obtaining fingering less typing skill helped by graphic conditioned response. After a decade of practicing with the skill, a trial succeeded in enhancing the aid to a visual aid for obtaining eight-fingered typing skill. Even school children with literacy can obtain the superior typing skill effortlessly and shortly. To the contrary of recent remarkable progress in PC capabilities, the general public worldwide has been without any typing methodology ever since invention of typewriter. The cause of it has existed in unbelievable difficulty of finding a clue to developing such an aid. The developed aid is ready to contribute to bridge the gap between PC and the general public. The aid would help fresh general public once a year and even eternally, since ideas, thoughts and others in the human brains are the last thing to be typewritten automatically.
\end{abstract}

Keywords: Multi-fingered typing aid; Visual eight-fingered typing skill; Bird's eye view of the keyboard; Graphic conditioned response; Finger assignment principles

\section{Introduction}

While working as an IT instructor for an internationally well-known computer company in Japan, I encountered heavy difficulty on a newly designed local PC (personal computer) keyboard. I created a graphic reference table, which finally reached a visual aid for obtaining eight-fingered typing skill.

Since the research was forced to start accidentally, it went without ascertaining nature of the research or without establishing a purpose, schedule or organization of it. When I was aware of it, the first half of the research had substantially passed away. Reflectively, purpose of the research should have been development of visual aid for obtaining eight-fingered typing skill. Due to lack of research planning and technological perspective, it took more than a quarter century to reach the final result.

The total story started when I encountered an accident on the PC keyboard. The accident forced me to cope with it urgently. It was caused by a large number of character keys (i.e., keys other than control keys) on the newly designed PC keyboard.

Modern PC appeared at the beginning of 1980s provided with locally designed keyboard which brought about a first chance for them to type sentences in native language. New-born 
Japanese PC keyboard was a hybrid one provided with both native-lettered key-set for primary native-key typing and alphabet key-set for secondary native key typing (i.e., foreign-key typing). The alphabet key-set was also used for typing in American English language. Apparently there was a big difference between the primary and the secondary typing method. When they selected primary native-key typing, they could concentrate on striking nativelettered keys. Unfortunately, the native-lettered keys threatened a calamitous key-search difficulty caused by randomly arranged as many as 46 native keys on the keyboard. As for secondary native-key typing, they had to search target keys among only 20 alphabet keys. However, twice as many alphabet keys must be struck for the makeshift secondary typing method. Additionally, translation was inevitable from a native input character to a couple of alphabet keys. Most of the young adult people did not worry anything about it. They seemed to select secondary one without fail. This selection sowed the seeds of future typing problem. I selected primary one. This selection brought about strange accident immediately after entering the first typing job on that day.

\section{Methodology}

\section{Opening with a Nervous Accident}

One day in 1986, brand-new huge PCs were delivered to the office, an education center of a computer manufacturer. I had a planned job of transcribing a draft for the next week. On Monday afternoon, I started the first typing job in native language in an office room with very few instructors inside. As anticipated, it took average 20 seconds to search each target key on the keyboard. Input characters came into PC keyboard disorderly whereas randomly arranged keys on the keyboard were ought to be searched thoroughly according to "left-to-right, up-todown" rule. When about a quarter minutes had passed, I felt the eye became blurred; then the eye and the neck were suddenly restrained from moving and would not move any more. The eyes were fixed at the central part of the keyboard. I became unable to continue the job. In a serene atmosphere of the room, I felt as if something invisible were monitoring the room. I was quite at a loss what to be done. Additional few minutes passed away slowly and silently.

Something invisible and I were expecting together something to happen. When I happened to be aware of the forehead getting feverish, I dared to stand up from the chair to leave the job. The restraints were released all at once. On the way home, I was thinking regrettably, "Even a slice of information about the target key location would be of great help."

\section{A Powerful Graphic Reference Table}

Next morning, before getting out of bed, "a slice of information" about the target key location was growing. On my way office, an image of a bird's-eye view of PC keyboard (hereafter, BEVK) was in my mind. In the office I created a graphic reference table containing 46 indexed BEVKs. Then, all the couples of index letter and BEVK were arranged orderly for quick reference. Finally, corresponding target key on each BEVK was colored in black. I tested the BEVK on the way. It guided me to the point straight (Figure 1). 


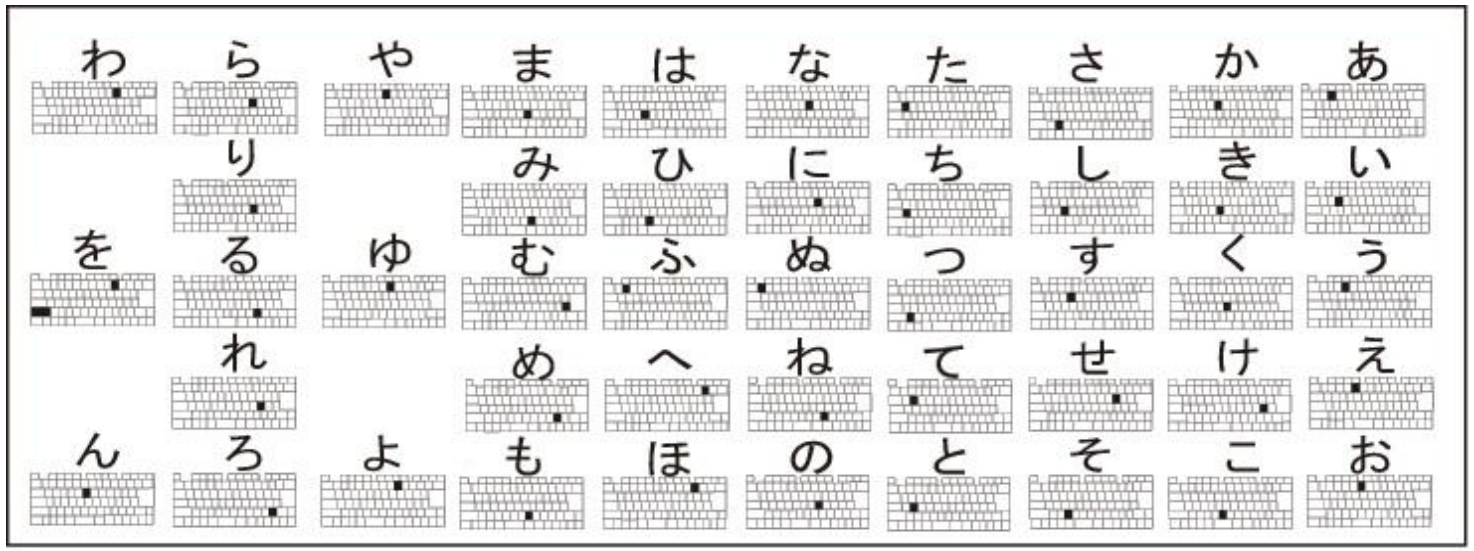

Figure 1. Specialized Graphic Reference Table

In Figure 1, couples of index letter and BEVK are arranged orderly for quick reference. Each black key denotes target key location. It helps learners find key locations easily. Several times of referencing makes learners learn key locations by heart accurately and unconsciously.

I was astonished to find the world had changed utterly from the previous day. Immediately after glancing at the black key for seizing it within BEVK, I could pinpoint the target key straight on the real keyboard. I thought that the reference table turned to pinpointing device for the target key. Additionally, while encountering the same character several times, graphic conditioned response was formed for it gradually. After completion of the formation, the eyes were made hopped reflectively onto the target key location at the sight of the character in the input stream. With the double surprise, the job progressed acceleratedly. A number of key locations memorized unconsciously increased day-by-day. Every morning, draft for the day was handwritten. In the afternoon, the draft was transcribed on PC with the help of the graphic reference table. All of the 46 key locations were memorized unconsciously and the transcribing job finished in less than four half days.

\section{Reflecting the First Half of the Research}

Reflectively, it was the very first day of typing in native language which had been longed for quite a long time in this country. In spite of it, I was knocked down by the calamitous keysearch difficulty. When I was leaving the room I heard something invisible muttered repeatedly, "If someone were attending me to foretell me the key location I am going to type next." I repelled the unrealistic suggestion. Something invisible may not have had more effective means of saving me other than restraining movements of the neck and eyeballs. The graphic reference table (or BEVKs, to be exact) worked far more than expected. Actually, it was enhanced to an aid for obtaining eight-fingered typing skill in the second half of this study.

\section{Beginning the Second Half of the Research}

PC installation to primary and secondary school started in 1998. At primary school they were regulated to learn primary native-key typing without any help for learning it. At secondary school, they had to learn secondary native-key typing discarding barely learned primary nativekey typing skill. One day in 2001, I happened to notice a primary school child (a granddaughter 
of mine) striking keys quite slowly with a single forefinger. I feared if the school children were going to follow my wake in 1986. Actually, child depression was spreading in parallel with the second half of the research going until 2008. The cause of child depression has not been discussed publicly or made clear even by the survey team of a certain Japanese university (Denda, 2008).

I hurried to incorporate a fingering facility into the inherited typing aid. At the same time, I began to think that the typing aid under development should have the possibility of being applied to the general public including school children with literacy. The second half of the research resumed from this point.

\section{The First Trial of Incorporation}

At first, I regarded incorporating fingering facility as an easy task. Hundreds of personified hand-shaped items were drawn to evaluate the legibility of them. Three kinds of information were used for denoting active hand (right or left), striking finger and target key location. As a result, it proved too be difficult to memorize different kinds of information located separately. I used to forget part of them during conveying them from BEVK to the real keyboard. The first trial was discontinued in a year.

\section{The Second Trial of Incorporation}

It was found by chance that a small circle placed on the target key location in the BEVK was not obstructive for locating the target key. Then I drew a small circle to be placed on the target key for denoting its location. Other necessary information was pushed together into the circle.

My wife who had been kept PC at a distance from the beginning suddenly wished to make an address book providing 50 entries. I handed her the result of the second trial with some instructions for typing addresses and few others. She completed it in three half days without querying about locating target keys. However, she gave up learning fingering on the way. I reflected that for a temporary typist, learning fingering seemed to be only a bothersome effort.

At any rate, effect of conspicuous denotation was confirmed at this trial. In addition to it, a part of beginner's common attention could be noticed.

Reflecting the result of the address book, I mailed directly to 2000 school masters and principals in Tokyo metropolitan area advertising the result of second trial, in vain. I also mailed it to more than $\mathbf{4 0}$ Local Education Committees. No response came to me, either. From this result and others, I had to feel something concealed or hidden within educational organization including responsible administration. I decided to give up the second trial. Then I began to think of advertising the research from abroad.

\section{The Third and Final Trial of Incorporation}

After failing twice, I wished to know touch typing methodology rather deeply. Since touch typing was based on "non-visual training" completely, I could not obtain any philosophical or descriptive documents at all. From an introductory book of touch typing, nothing suggestive 
was gained except a title less and page-numberless piece of chart inserted in the book. The chart can be seen broadly in the related site of the Internet. It was supposedly drawn by the inventor of touch typing in 1880 s.

At a glance, the primitive chart impressed me calmly. The chart seemed to have been drawn for showing principle of finger assignment on the keyboard (hereafter, the finger assignment principle). I considered that drawing hand postures was the most urgent and indispensable task for the incorporation. The task started immediately modeled on own hand. I began to draw hand postures at the moment of striking target key in each BEVK. Drawing/amending was quite a time-consuming task spending nearly four years. It is commonly heard that only expert painters should be able to draw human hand precisely and lively.

To return to the subject, the drawing/amending task produced fruitful results. At nearly end of the task, I could deduce three hidden principles from the drawings; "Individual finger should be responsible for predetermined keys nearby" and "Individual key should be struck with predetermined finger nearby" and "Every finger must be able to type any responsible keys without horizontal/vertical hand movements." I sublimated the hidden principles to rules of "Discrete finger movement" and "Flexible finger movement", which will be mentioned later.

I confirmed that for incorporating those principles into the developing typing aid, hand postures and BEVKs were most appropriate vehicles for accepting them. As a result, Figure 1 was improved to Figure 2 below. This concluded the third trial successfully.

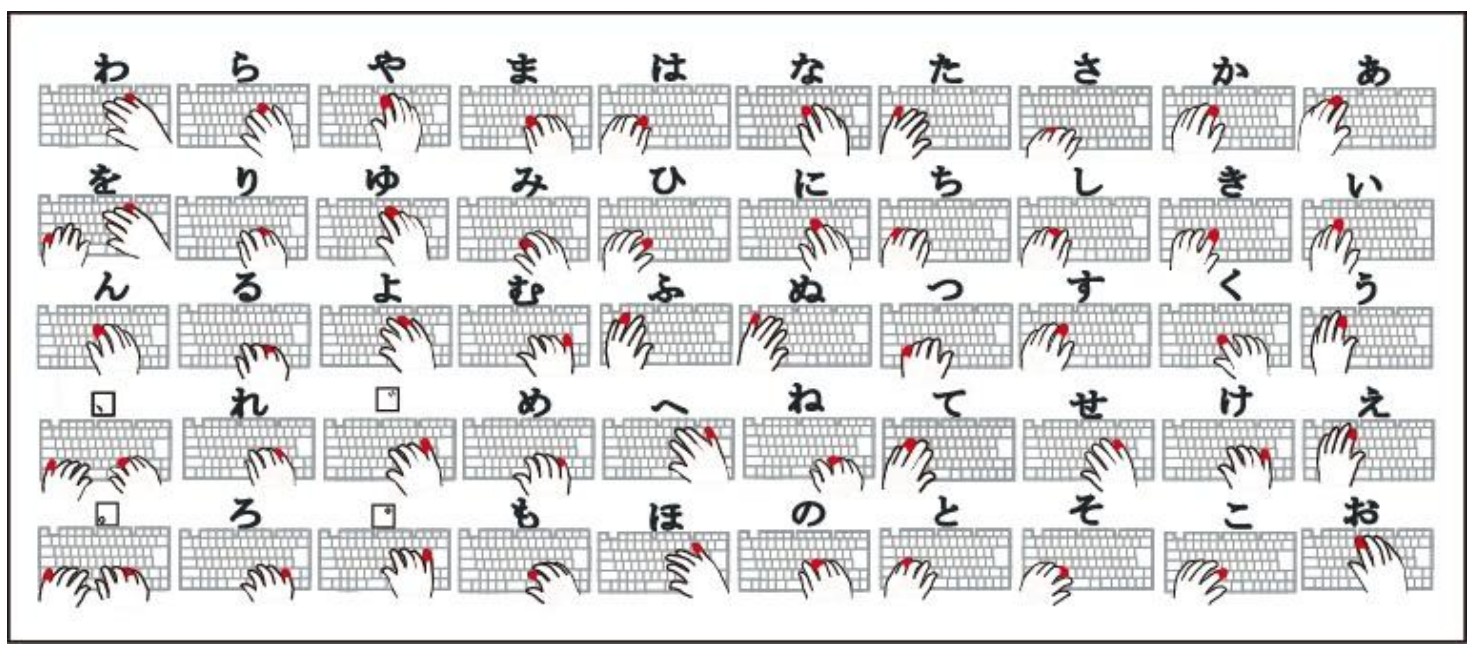

Figure 2. Innovated Graphic Reference Table

Figure 2 above shows a visual typing aid for obtaining eight-fingered typing skill. It contains both pinpointing and fingering facilities. Hand postures take charge of fingering facility. Target key location is a base of pinpointing facility. Thus the initial graphic reference table has been innovated to eight-fingered typing aid in 2008.

\section{Findings}

The most vivid and helpful encountering with something invisible was at the beginning of the study. Long after that I came to consider it to be the nerve center within me. Now that it is hierarchically highest nerve system, it could control peripheral nerves and muscles safely. 


\section{Results}

\section{A Long Journey to Eight-fingered Typing Aid}

More than two decades had passed since a device was created for reducing the scope of the calamitous key-search difficulty. The reducer developed in 1986 has been innovated to a visual aid for obtaining eight-fingered typing skill (hereafter, Vaid-8). With the help of the aid, even school children with literacy can obtain the skill through transcribing exercise (hereafter, the exercise) shortly and comfortably. It also actualized the possibility of spreading visual eightfingered typing skill worldwide.

\section{Structure of Vaid-8}

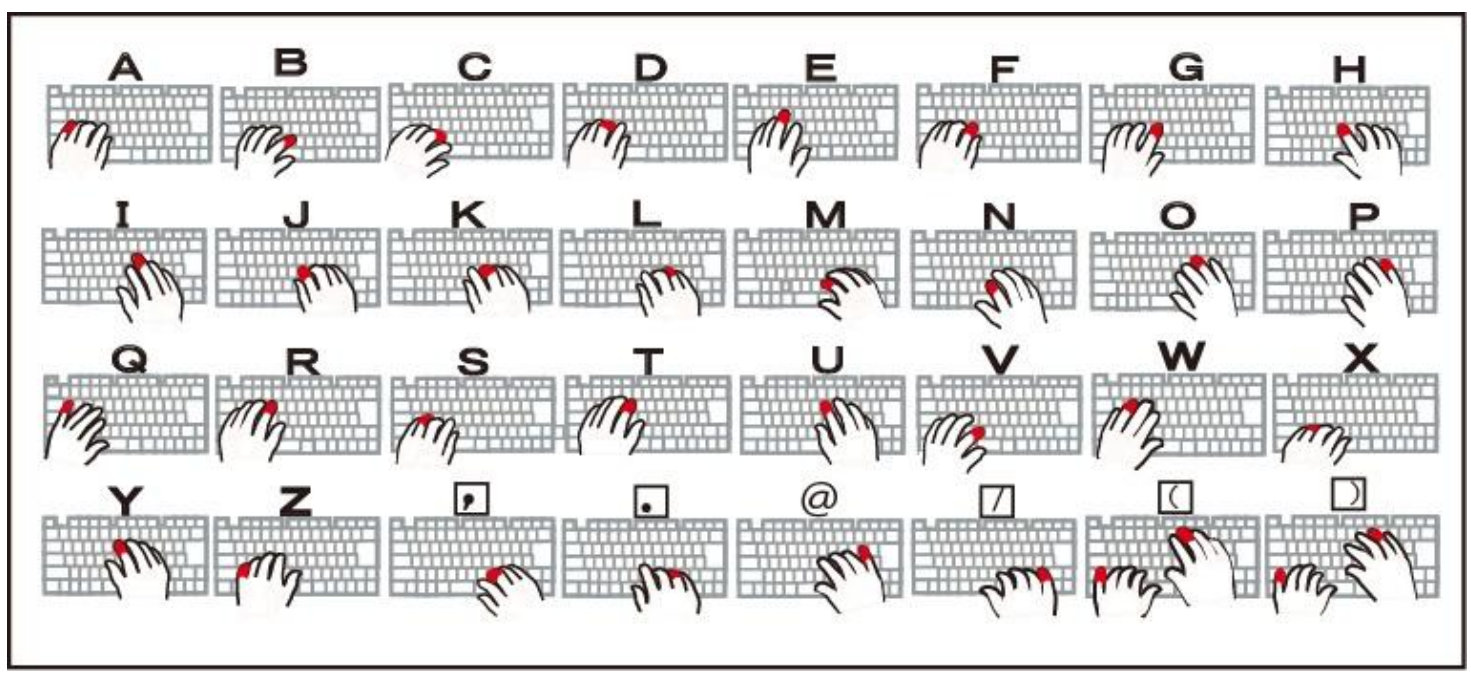

Figure 3. Vaid-8 of U.S. Version

Figure 3 shows Vaid-8 of U.S. version. Vaid-8 is internationally available through simple customization. In this Figure, BEVKs are seen inherited from the initial typing aid (i.e., graphic reference table). Index letters on each BEVK show the same character is to be typed when corresponding target key is struck. Black keys seen in Figure 1 have been replaced by hand postures. Hand posture shows the figure of hand at the moment of striking corresponding target key. Idle hand postures have been expelled from Vaid-8 for clarity. Individual hand posture is unique in shape. According to the finger assignment principle, each key is struck by predetermined and stretched/bent finger with a fulcrum of the wrist.

One indexed couple (i.e., hand posture and BEVK) is shown enlarged in Figure 4. A red-fingertip (hereafter, red-tip) in hand posture can denote both striking finger and target key location because striking finger is piled just on the target key for easier operation in the exercise. This is also for prevention of ignoring striking finger. Beginners are attracted to target key location more than striking finger. This piling up is preferable because striking finger and target key location are on friendly terms. In addition to it, it is valid because target key location must be identified absolutely whereas striking finger can be denoted anywhere within the same BEVK. Figure 4 tells learners that the target key under the red-tip should be struck by the red-tip (i.e., striking finger). 


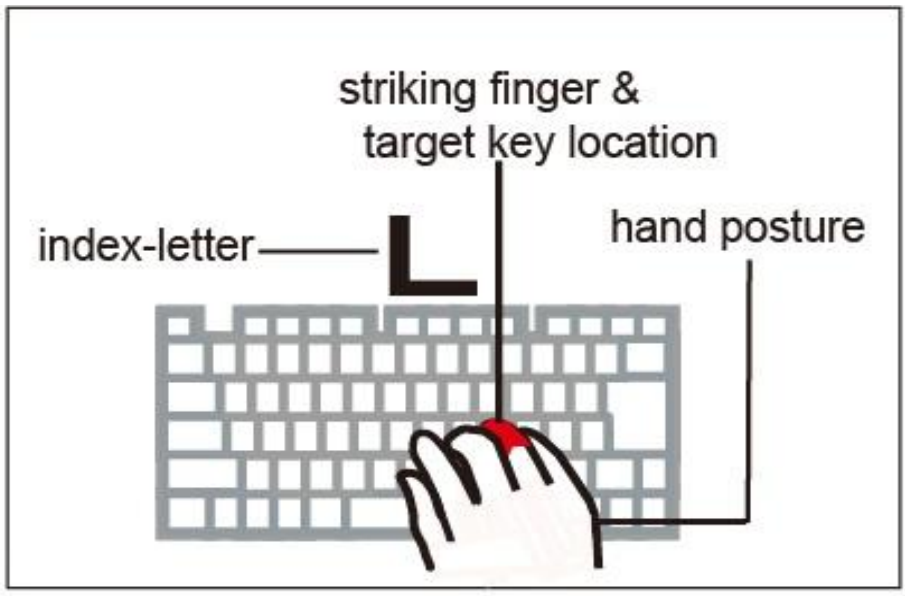

Figure 4. BEVK of U.S. Version (enlarged)

\section{How to Learn Eight-Fingered Typing Skill}

\section{Basic Procedure}

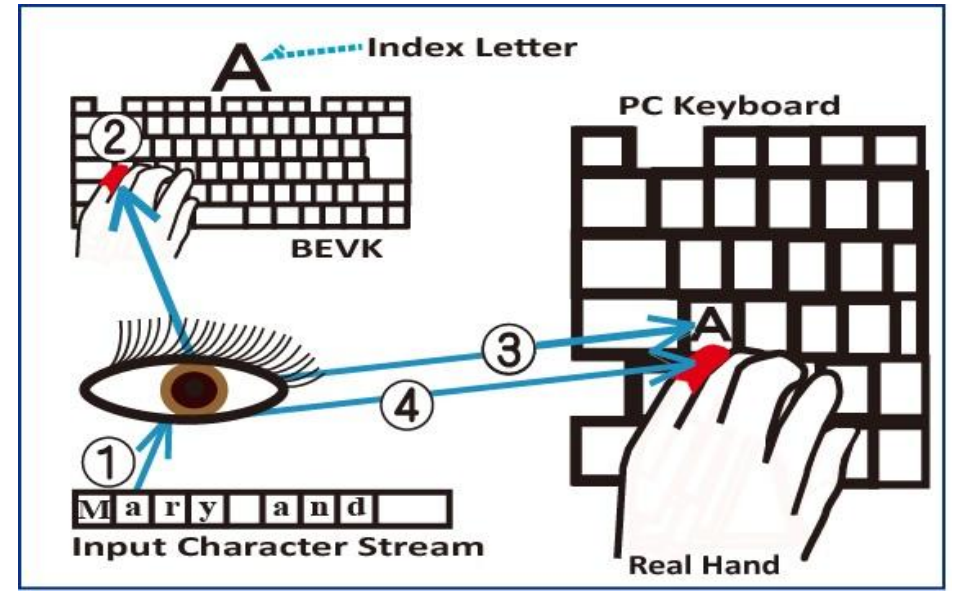

Figure 5. Four Steps of Consulting

The eight-fingered typing skill is learned through 4 steps of consulting as shown in Figure 5. The following corresponding steps are executed serially for each character in the input stream (or in mind.)

(1) Take up next character to be typed. Find corresponding index letter.

(2) Look at BEVK for seizing the location of red-tip on the real keyboard.

(3) Hop onto the target key on the real keyboard as imitating the hand posture.

(4) Find and strike the target key with real red-tip (i.e., with real striking finger) 
While repeating the consulting several times, the four items (i.e., index letter, striking finger, target key location and active hand) can easily and firmly be memorized with the help of graphic conditioned response and the following attributes.

- Coordinated denotations within hand shape

- Piled up items (striking finger and target key location)

- Associative and real-shaped items (hand posture and fingers within it)

\section{Formation of Graphic Conditioned Response}

During repeating the consulting several times, all the steps (1) - (4)can be memorized firmly. Time needed for all of them is reduced accordingly. When the time for steps (1) - (3) reduced to nearly zero is the completion time of forming the graphic conditioned response. After that, at the sight of the character (at step (1)), the eyes are reflectively made hopping onto the target key location at a bound (at step(4)) with necessary information firmly learned by heart. The target key can be struck at step (4).

After all the graphic conditioned response has been formed in the same way, learners can obtain eight-fingered typing skill as an outcome of the exercise. From my experience, the newly obtained typing skill showed speed of five characters per second, though it was done at rare intervals. (Time depends on individual differences and number of character keys on the keyboard.)

Adding a word to it, graphic conditioned response is a very common and popular phenomenon in our daily lives. For instance, 'I remember seeing him once.' is an outcome of a simple graphic conditioned response. Reading a part of a text repeatedly is a means of memorizing it by heart exploiting graphic conditioned response, though some degree of individual differences may exist among a group of people.

\section{Whole Process of creating a New Typing Skill}

Necessary time for passing the 4 steps is gradually reduced to zero when graphic conditioned response is being formed for the characters. Current length of time necessary for forming a graphic conditioned response changes dynamically according to the ratio of appearing of each character in the input stream. At any rate, a character whose necessary time reaches zero gains privilege of graphic conditioned response. In that way, number of privileged characters increases as the exercise goes on. When all the characters have gained a graphic conditioned response is the time of completion of the exercise for a character set (Figure 6). It is not mandatory for a learner to understand the whole process and mechanism of graphic conditioned response. He or she should only do the exercise steadily and comfortably for obtaining eight-fingered typing skill. 


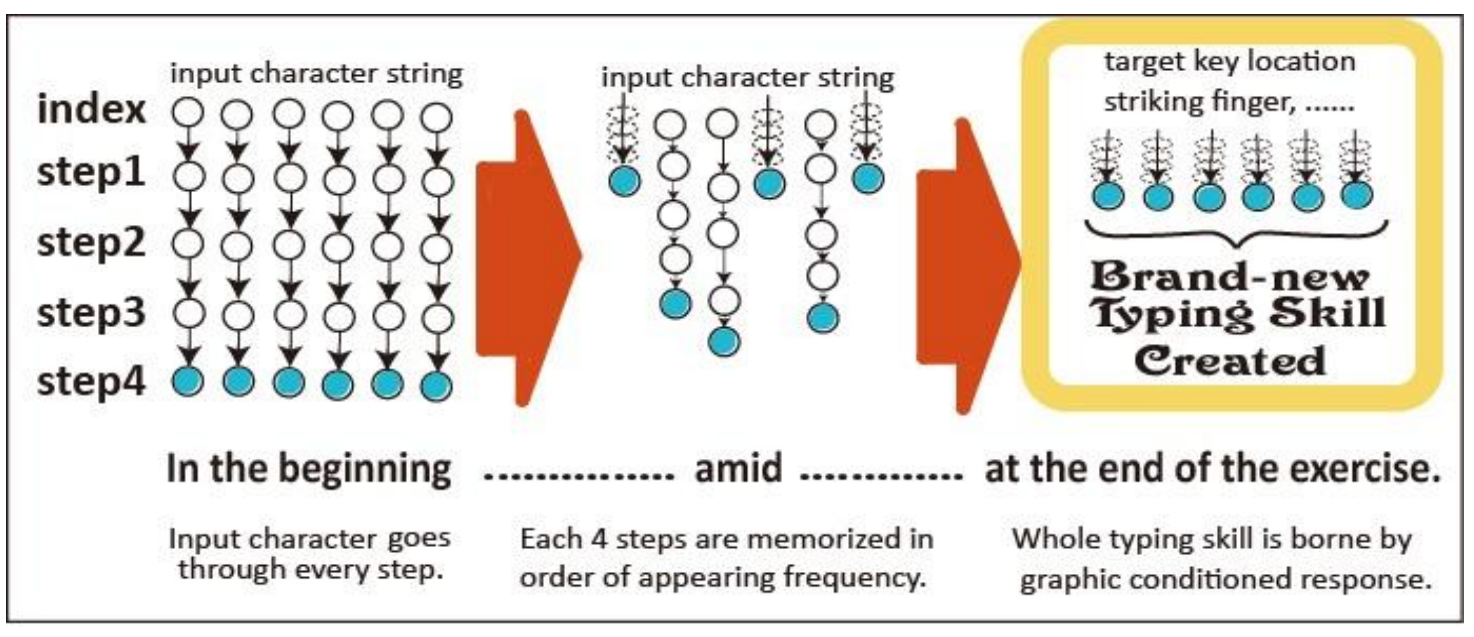

Figure 6. The Exercise and Its Outcome

Therefore, a graphic conditioned response may be called as a slice of new typing skill. When whole the graphic conditioned responses for a character set are formed, it is the time for a post-learner to obtain a new entire typing skill.

\section{Source of Visual Typing Efficiency}

While typing, hand movements should be expelled from learners' typing for the sake of rapid and accurate typing. Discrete finger movement expels horizontal hand movements by way of the finger assignment on the keyboard. Flexible finger movement expels vertical hand movements by stretching/bending striking finger to reach the target key properly. These desirable finger movements influence hand postures without fail. Accordingly, learners can obtain the desirable finger movements during the exercise naturally and almost unconsciously.

In visual typing, it is usual to confirm a target key-letter just before striking it. The confirmation contributes to typing accuracy and thereby to reducing correction time of typing errors. The two kinds of finger movement together with confirmation of accurate typing form a firm basis for pursuing ultimate typing speed and accuracy in visual typing.

\section{Capability of Vaid-8}

While transcribing some document with the help of Vaid-8, even brand-new typing beginners can obtain eight-fingered typing skill shortly, effortlessly, willingly and comfortably.

Learners can learn entire typing skill (i.e., pinpointing and fingering skills) through the exercise with the help of Vaid-8. The exercise makes it easy to learn typing skill because everything is shown graphically or picturesquely except index letter. Graphic conditioned response helps learners to learn typing skill easily, shortly and effortlessly. So they can do the exercise with little help of human teachers or without hard effort of memorizing key locations and its fingering. The intuitive and guessing nuance (i.e., locating and hopping) during the exercise encourages learners to do the exercise willingly and comfortably. It takes them net 3-6 hours to finish the exercise. (Time depends on the number of character keys registered on Vaid-8 and on individual differences.) After finishing the exercise, post-learners can type rapidly, 
accurately and continuously owing to obtained pinpointing skill and fingering skill leaving off Vaid-8 of course. (School children with smaller hands may not cover some remote keys. Small movement of hand(s) while typing is allowable for them until time resolves the difficulty).

\section{Eternal Partnership}

To the contrary of recent remarkable progress in PC capabilities, the general public worldwide has been without any typing methodology ever since invention of typewriter in 1880s. The cause of it lies entirely on the difficulty of finding a clue to developing multi-fingered typing aid. The fortunate Vaid-8 is ready to contribute to bridge the gap between the general public and PC. Vaid-8 would be with the fresh general public yearly and even eternally because ideas, thoughts and others in the human brains are the last thing to be typewritten automatically.

\section{Outcomes of Vaid-8 International}

Depending on the type of the keyboard in use, attitude of the general public would be varied at the news of Vaid-8.

Category-1: This category should be an overwhelming majority in the world. Where the general public using keyboard provided with moderate number of alphabet keys traditionally, most of them may have been accustomed to pseudo multi-fingered typing. Vaid-8 should be welcomed after they have noticed school children type making showers of fingertips on the keyboard. Some nation in this category with more than 30 character keys, may have longed for a superior visual typing method. They may show quite different attitude at the news of Vaid-8.

Category-2: A Far East island country falls into this category. Nearly all the national people who escaped from primary native-key typing disappointedly in 1986 will be wild with joy at the news of Vaid-8 (hopefully).

Category-3: Another Far East country and areas with ideograms solely fall into this category. The general public with native non-alphabet keyboards will also enjoy the effect of eightfingered typing, although they are unable to overcome the restraint of enormous number of ideograms at this time, too. (They already experienced trials in 1980s.)

Category-4: Nations, whose PC use are limited to government agencies and large organizations currently, will take some time until the exercise is fully incorporated into the curricula of compulsory education.

\section{Potentiality for the General Public}

The general public occupies most of the population of a country and/or area(s). Therefore, they essentially support countries or areas where they live in. Many of them are working in a variety of fields of business or administration where PC is used as indispensable tool. If their untutored typing is enhanced to uppermost typing technology shortly, they will be able to act much more effectively and efficiently in their businesses and private lives. The more volume they type with higher quality, the more merits will be enjoyable. Large amount of saved time will be at their discretion supposedly. 


\section{Comparison among Typing Styles}

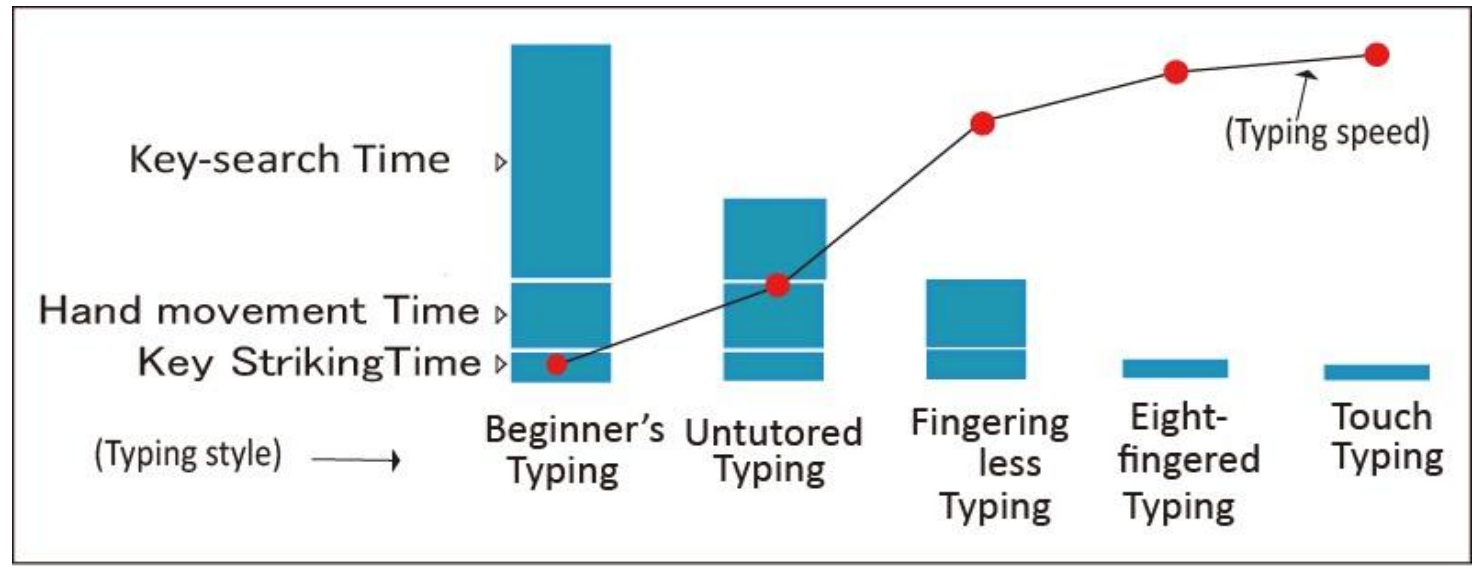

Figure 7. Typing Styles in Terms of Times and Speed

Beginner's typing is displayed by brand-new typing beginners who consume maximum typing time in key-searching and others. Hence, performance (speed and accuracy in typing) should be the lowest.

Untutored typing is commonly learned unconsciously by post-beginners. Nearly all of them have little memory of key locations. This typing style has commonly been seen among the general public in the world. Some of them display pseudo multi-fingered typing.

Fingering less typing was learned through the initial graphic reference table. This typing skill expelled key-search time completely. It worked pretty well more than a decade until enhanced to eight-fingered typing.

Eight-fingered typing displays touch typing-like performance, for fingering principles of touch typing has been introduced into Vaid-8. Owing to optic nerve, visual typing skill can be learned shortly, effortlessly and comfortably during the exercise.

Touch typing provides most superior typing skill obtained through minutely designed blind touch training. Every finger is trained so as to reach any keys rapidly without making use of optic nerve at all. Touch typing people say that own fingers can type autonomously as if eyes were on each of eight fingers.

Above comparison is done based on pure typing time excluding impure typing time such as thinking time or instant elaboration time. Thinking time is usually indispensable in most of the typing works done by the general public whereas thinking time in stenographic work, for instance, would be restricted to a minimum.

\section{Hopeful Candidates of Vaid-8}

Primary school children with literacy are hopeful candidates of Vaid-8. First of all, they are yet free from untutored fingering. Their brains and fingers are young and elastic. Most of them are 
interested in PC and are full of curiosity. They may need less time for the exercise. If the exercise is planned to be added to a curriculum of compulsory education, they will surely be making a new member of literacy, namely typewriting.

\section{Elaboration after Composition}

Elaboration should not be underestimated especially for child learners. During elaboration of own writings, reading, amending and composing ability is stimulated and polished. As a whole, elaboration is a powerful tool for improving quality of writings as is known widely. Young people should learn more while elaborating their writings with the help of modern powerful editing functions of PC software. After all, their typewriting literacy will be enhanced remarkably in the long run.

\section{Customizing Vaid-8}

Vaid-8 can be customized using Skelton Model (Figure 8). Customized Vaid-8 is needed for each unique keyboard model in use. It is done regarding index letters and their arrangement on Skelton Model. Number of Special Characters to be registered on Vaid-8 is optional. It is not recommendable to include all of the Special Characters without considering real usage of them.

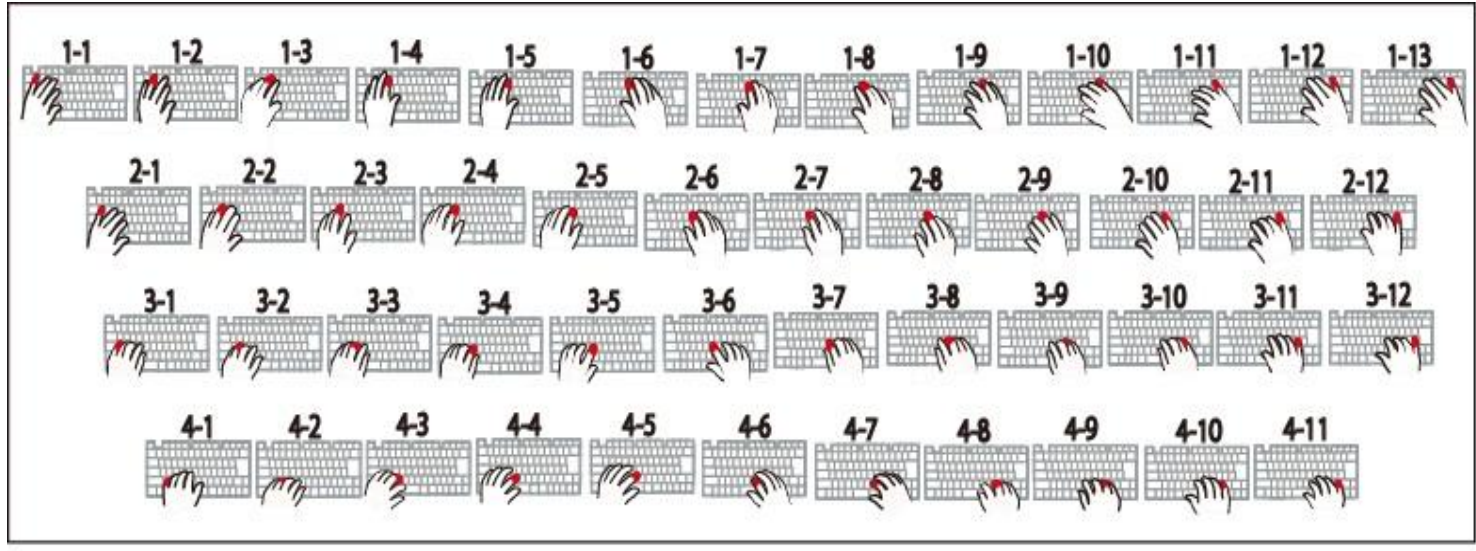

Figure 8. Skelton Model for Customization

For customizing Vaid-8, an electronic copy of Skelton Model is used. Customization is done by replacing coordinate's numerals on the Skelton Model with corresponding index-letters of the keyboard in use. A Skelton Model is needed for each type of the keyboard. In any case, hand postures must be kept unchanged. (The Skelton Model was drawn based on Figure 2. Adobe Illustrator is used for drawing, electronic copying and customizing Skelton Model.)

\section{For the People with Untutored Fingering}

Most of the adult people have been unable to get out of untutored fingering by themselves. They are now able to do it with Vaid-8. A knack in doing it is as follows. Slowly but steadily doing the exercise is an ideal way of it. Anyway, the result of it may come earlier than expected. 


\section{For the Handicapped}

Vaid-8 may be used for obtaining only pinpointing skill. This typing skill is obtained through the exercise ignoring fingering denotations altogether. Sole pinpointing skill acquired will contribute to considerably rapid and accurate typing. Single-handed typing can be learned through the exercise using only single active hand. All the hand postures should be regarded as belonging to the single active hand with/without fingering. Even with leg(s) or with a stick in his or her mouth, pinpointing skill without fingering may be learned through the exercise in its own way.

\section{The Exercise}

\section{The Principle of the Exercise}

The exercise is the most efficient way of obtaining eight-fingered typing skill. It takes 3-6 hours in total depending on the number of character keys on the keyboard and on individual differences. Actually, "to learn typing skill" does not mean here "to be given typing skill" but "to obtain typing skill willingly and comfortably". Willing attitude is fostered naturally during the exercise.

\section{Preparing for the Exercise}

It is advisable that an exercise is to be divided into sessions of 20-30 minutes each followed by a break. Any familiar document can be used for the exercise; text pages, newspapers, magazines and so forth. When using printed Vaid-8, PC should be prepared for transcribing some document into work area where elaboration can be done.

\section{Key Procedure of the Exercise}

Key procedure of the exercise is Main step preceded by Pre-main Step-1 and 2. Both open hands are over the keyboard and both wrists are beside the keyboard holding Space Key and/or equivalent between them. Be sure red-tip denotes both striking finger and target key location. Keys are to be struck lightly and calmly and rapidly, if possible.

(Pre-main Step-1) Read word(s) on the transcribing document. Keep them in mind.

(Pre-main Step-2) Take up one character to be typed.

(Main Step) Find corresponding index letter. Look at BEVK for seizing the location of red-tip on the real keyboard. Hop onto the target key on the real keyboard as imitating the hand posture. Find and strike the target key with real red-tip (i.e., with real striking finger).

When the fingering or target key location is forgotten on the way, the main step must be redone much more slowly than usual. If input character exhausted, go to Step-1. Otherwise continue from Step-2. 
When completed the exercise ignoring 'as imitating hand posture' above, inappropriate stretching/bending will be seen here and there. It will disturb typing efficiency by hand movements brought about with the ignorance.

After learned by heart a target key location together with its fingering, learner's eyes are suddenly popped onto the real target key location straight at the sight of the character to be struck in the input stream (or in mind). This has been the outcome of graphic conditioned response. The target key can be struck immediately. Number of such keys will surely increase and the exercise is accelerated accordingly.

Usually at the beginning of a session or of a day, slower starting speed is most preferable. The typing speed will soon be accelerated naturally beyond usual speed. The exercise should be continued until the end of a session or until the entire target key locations with fingering are memorized by heart. Most realistic means of improving typing speed should be to have a plenty volume of typing at times. Most effective way of improving typing quality should be elaboration at any moment.

\section{Joyful Presents for Learners of Eight-Fingered Typing Skill}

At nearly end of the exercise in American English language, I was given joyful spiritual presents in the brain. One of them was an imaginary short and thick woolen string in dark blue. During the very slow movement of the string from right to left in the brain, I perceived indescribably pure and serene feeling for several seconds. At the secondary encounter with it, a couple of days later, I could perceive its coming beforehand, because the string was taking time in preparing for the appearance in the brain. The same show was enjoyable as before.

Another kind of present experienced was a show of fingertips on the keyboard. When fingers were working discretely and flexibly, they were seen displaying showers of fingertips on the keyboard. The showers of fingertips may be seen at near end of the exercise probably more than several times in a period of time.

\section{Learning Additional Typing Key-Set}

After learning a typing skill on a certain keyboard perfectly, another key-set can be learned on the appropriate keyboard without confusion. On the contrary, concurrent learning of different key-sets on the same/different keyboard(s) should be avoided because of confusion.

\section{Vaid-8 in e-Learning Environment}

Initial Vaid-8 was printed on a sheet of paper. Modern Vaid-8 could work online much better when provision for it is made by PC software manufacturer. Figure 9 is just an example of Vaid8 in e-learning environment. 


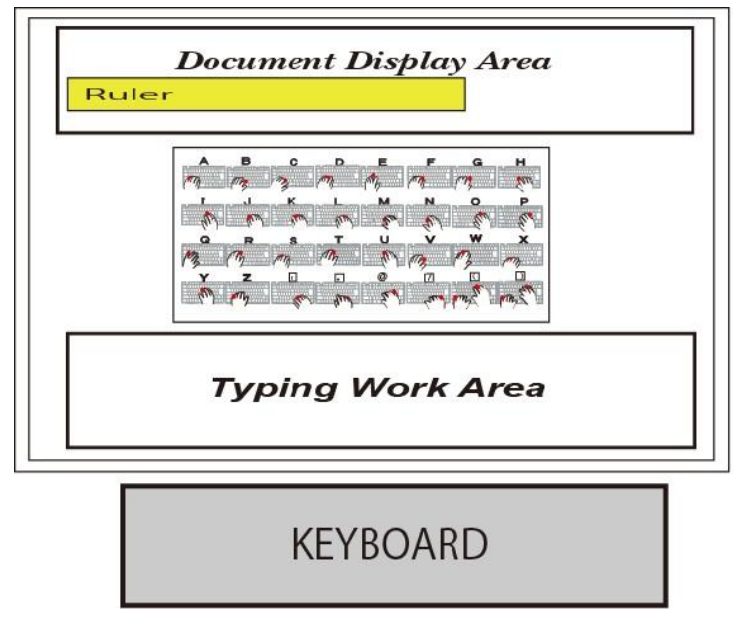

Figure 9. Example Screen Layout for Vaid-8 online

Document Display Area is for document data to be transcribed in the exercise. It would be convenient if replaceable and scrollable. Ruler is for reading convenience. Vaid- 8 should be displayed on the center of the screen. The Vaid- 8 should be selectable and replaceable on the screen according to the keyboard currently in use. Characters typed in go into Typing Work Area. Contents in this area should be editable and scrollable for elaboration. Real keyboard is placed on normal position.

\section{Conclusions and Recommendations}

By means of recognizing BEVK as basic elements of fingering less typing aid, I could reach visual eight-fingered typing aid. For disseminating Vaid-8, many things should be prepared including data about the keyboard layout worldwide. People participating in the dissemination of Vaid-8 are to be acquainted with many things including educational situations worldwide, eightfingered typing technology, e-learning environment and others. For smooth, orderly and planned dissemination, advanced PC software manufacturer can be considered to be the ablest organization.

\section{References}

Denda, K. M.D. (2008). Depression in children and adolescents: From the viewpoints of developmental disorders and bipolar spectrum disorder (Unpublished scholarly paper). Department of Functioning and Disability, Faculty of Health Science, Hokkaido University. Sapporo, Japan. Available at: http://www.hs.hokudai.ac.jp/denda/

Correspondence: Junichi Watanabe, 2-4-9 Higashiiko, Adachi-ku, Tokyo 121-0801, Japan 